\title{
Particle-in-cell Monte Carlo simulation using free path method for radio frequency argon discharge modelling: Comparison with experiment
}

\author{
Djilali Benyoucef ${ }^{1 \star}$, Mohammed Yousfi ${ }^{2}$ and Bachir Belmadani ${ }^{1}$ \\ ${ }^{1}$ Laboratoire Génie Electrique et Energies Renouvelables, Chlef University, Algeria. \\ ${ }^{2}$ Laplace, UMR du CNRS 5312, Université de Toulouse, France.
}

Accepted 23 August, 2012

\begin{abstract}
A specific particle-in-cell Monte Carlo model which is based on an optimized free path method for collision treatment has been used to simulate a radiofrequency (RF) argon discharge at low pressure for parallel-plate geometry. In this model, the free path determination is independent of the electron energy; it depends only on the maximum of the total cross sections. The obtained results are in excellent agreement with experimental results taken from previous literature in the case of RF discharge in argon. Furthermore, the present model is faster than the classical one based on free time of flight method extensively used to study the low pressure electrical discharges.
\end{abstract}

Key words: PIC MC technique, radiofrequency low pressure discharge, free path for collision treatment.

\section{INTRODUCTION}

Collisions of charged particles (electrons and ions) with gas molecules are one of the key processes which govern the properties of non thermal plasmas generated by non equilibrium electrical discharges used for applications, such as material processing or deposition of thin films for manufacturing microelectronic devices or light sources (Sandu et al., 2006, Hellegouarch et al., 2001; Hess, 1996). The good knowledge of the discharge properties requires accurate models able to give quantitative description and prediction of the discharge characteristics. Modelling of electrical, physical and chemical characteristics of RF low pressure discharge (due to the high non equilibrium processes inherent to these low pressure plasmas) needs the use of particle models rather than the fluid models. It consists in studying a sample of particles represented statistically. The simulation of the trajectories of such particles requires the knowledge of the elastic and inelastic collision processes, which are represented by their cross sections. The probability of each kind of collision is

\footnotetext{
*Corresponding author. E-mail: ben_plasma@yahoo.fr.
}

determined from the integration of the corresponding cross section in the velocity space. Due to the huge collision number to treat, these integration which are performed numerically takes a long computing time. To improving the latter, Skullerud (1968) used a free time of flight method and introduced the "null collision" method where a fictitious process, without any effect on the particle trajectory, is added in order to have always a total collision frequency constant. Generally, the total collision frequency is chosen equal to the maximum collision frequency (Yousfi et al., 1994; Van der Velden et al., 2006; Wu et al., 2006; Kawamura et al., 2000; Brennan, 1991; Koura, 1986).

In this work we developed a free path method for the collision treatment. This method is then tested in the case of the simulation of an argon radio frequency glow discharge at low pressure. To show the speed of the present free path method of collision treatment, we compared the computation time to a classical free time of flight model. We also verified the validity of the present particle model and the accuracy of our results from comparisons of the calculated electron densities to the measured one taken from the literature. Last, electric RF discharge characteristics such as plasma potential and 


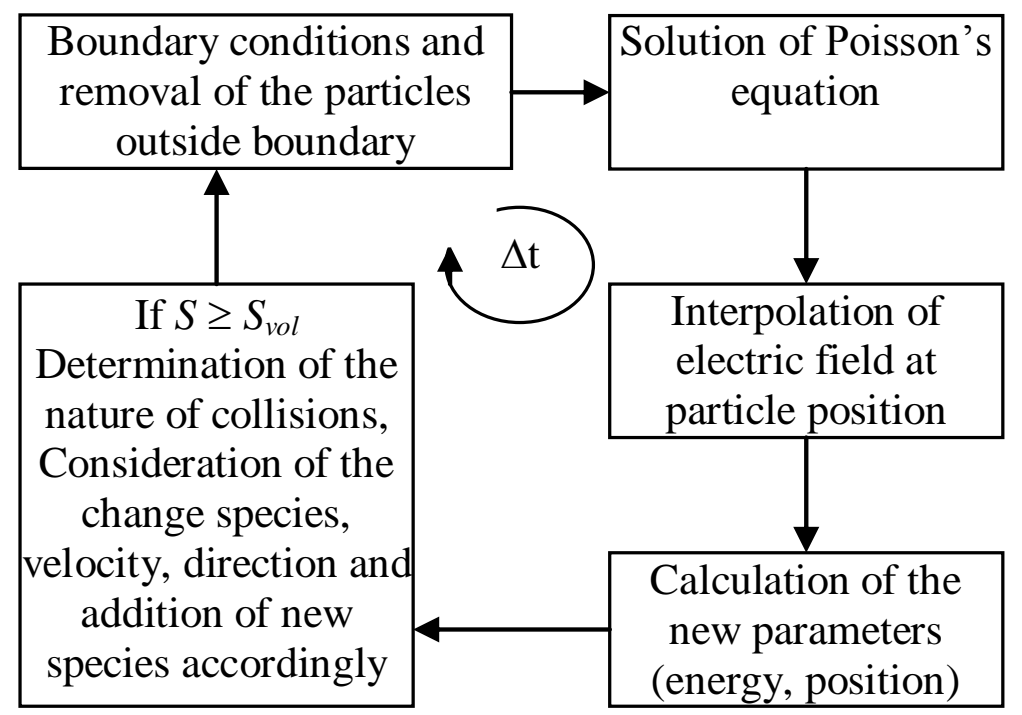

Figure 1. Flowchart of the required sequential steps. $S$, the distance covered after the last collision or the distance between two successive collisions $\mathrm{S}_{\mathrm{vol}}$, distance covered without collision.

electric field have also been discussed.

\section{METHODS}

\section{Description of collision treatment from free path method}

The discharge is simulated using a PIC MC code based on the estimate of the free path $\lambda_{v o l}$ which gives the particle position after every collision. Due to their large number, electrons and ions of argon are, as usual in particle method, considered as pseudoparticles. Each pseudo-particle represents a large number of real particles. The computational grid divides the space definition domain into a specific number of cells. Each cell must contain at least 100 particles to ensure good statistics. Concerning the time step, there are several criteria to choose it, one quotes for example criteria of Nanbu (1994) and Sugawara et al. (2007). In this work, the time step (used for the integration of the classical mechanics equations of the motion) is chosen lower than the mean free flight time. The flowchart given the sequential steps required during the simulation of the charged particle transport is shown in Figure 1.

To minimize the number of arithmetic operations, the space charge electric field is calculated analytically without numerically solving the Poisson's equation, this field at point $x$ is given by the relation:

$E(x)=\int_{0}^{x} \frac{\rho(x)}{\varepsilon_{0}} d x$

$E(\mathrm{x}), \rho(\mathrm{x}), \varepsilon_{0}$ : are respectively, the electric field, the net charge density at point $x$ and the vacuum permittivity. The integral is calculated according to the boundary condition $E(x=0)$, this condition must check the following equation:

$-\int_{0}^{d} E(x) d x=V_{a}-V_{c}$
$V_{a}$ is the anode voltage, $V_{c}$ the cathode voltage where we applied the radio frequency voltage and $d$ the gap spacing. The electric field to each node $(i),(i=1, . ., n)$ :

$$
\mathrm{E}_{\mathrm{i}}=\mathrm{E}_{\mathrm{i}-1}+\frac{\rho_{\mathrm{i}}}{\varepsilon_{\mathrm{o}}} \Delta \mathrm{x}
$$

where the electric field at the cathode is given by:

$$
E_{0}=\left(\frac{V_{c}}{\Delta x}-\sum_{i=1}^{n} E_{i}+\frac{E_{n}}{2}\right) \frac{2}{2 n+1}
$$

In order to obtain the evolution of the potential distribution, the electric field along the axis of discharge is integrated, for example, using Simpson's method to obtain the potential $V_{i}$ at each node $i$ of the mesh:

$\mathrm{V}_{\mathrm{i}}=\mathrm{V}_{\mathrm{i}-1}-\frac{\Delta \mathrm{x}}{6}\left(\mathrm{E}_{\mathrm{i}-1}+4 \mathrm{E}_{\mathrm{i}}+\mathrm{E}_{\mathrm{i}+1}\right)$ avec $\mathrm{V}_{0}=\mathrm{V}_{\mathrm{c}}$

In the presence of an electric field $E$, the charged particles (of charge $e$ and mass $\mathrm{m}$ ) are subject to the acceleration (a) of the Lorentz force $(\mathrm{ma}=\mathrm{eE}$ ) which obeys the laws of classical mechanics. The vector components of the position $(x, y, z)$ and the components of the vector velocity $\left(v_{x}, v_{y}, v_{z}\right)$ of the particle after a step in time $\Delta t$ are:

$$
\left(\begin{array}{c}
v_{x} \\
v_{y} \\
v_{z}
\end{array}\right)=\left(\begin{array}{c}
v_{x 0} \\
v_{y 0} \\
v_{z 0}
\end{array}\right)+\frac{e \Delta t}{m}\left(\begin{array}{l}
E \\
0 \\
0
\end{array}\right)
$$




$$
\left(\begin{array}{l}
x \\
y \\
z
\end{array}\right)=\left(\begin{array}{l}
x_{0} \\
y_{0} \\
z_{0}
\end{array}\right)+\left(\begin{array}{c}
v_{x 0} \\
v_{y 0} \\
v_{z 0}
\end{array}\right) \Delta t+\frac{e \Delta t^{2}}{2 m}\left(\begin{array}{l}
E \\
0 \\
0
\end{array}\right)
$$

$\left(\mathrm{x}_{0}, \mathrm{y}_{0}, \mathrm{z}_{0}\right)$ and $\left(\mathrm{v}_{0 \mathrm{x}}, \mathrm{v}_{0 \mathrm{y}}, \mathrm{v}_{0 \mathrm{z}}\right)$ are the initial position and velocity of a given particle, respectively.

In this study, only the collisions between the charged particles and the neutrals are taken; because the plasma is weakly ionized. Considering that a charged particle has $N$ types of collisions, the total cross section can be obtained from the following relation:

$$
\sigma_{T}(\varepsilon)=\sum_{i=1}^{N} \sigma_{i}(\varepsilon)
$$

$\varepsilon:$ is the kinetic energy of incident particle and $\sigma_{i}(\varepsilon)$ : is the collision cross section for the process $\mathrm{i}$ (elastic or excitation or ionization). The free path $\lambda_{\text {vol }}$ between two successive collisions depends on the background gas density $n(S)$ at the position $S$ and the energy $\varepsilon$ of the incident particle.

$$
\lambda_{v o l}(\varepsilon)=\frac{1}{n(s) \cdot \sigma_{T}(\varepsilon)}
$$

If $P(S)$ is the moving probability of the particle without collision, the collision probability at the position $S$ is given by the following relation:

$$
1-P(s)=1-\exp \left(-\int_{0}^{s} n(s) \sigma_{T}(\varepsilon) d s\right)
$$

$S$ is the distance covered after the last collision or the distance between two successive collisions. To determine this distance $S$ (upper bound of integral over space) where the particle undergoes a collision, it is necessary to know the collision cross section after each time step because the energy of the particle changes after the collisions due to the electric field action. Therefore, we must integrate the relation as presented in (10) along the trajectory of the particle which necessary requires some computing time because the solution of equation (10) is not analytic but numeric due to the complex variation of cross section versus energy. To avoid this computing time problem, we considered a minimal and constant free path $\lambda_{\min }$ from the choice of a constant and maximal total collision cross section $\sigma_{T, \max }$ that involves real collisions (elastic, excitation and ionization) and also an additional fictitious collision whose magnitude is chosen to have a total collision cross sections constant whatever the energy:

$$
\lambda_{\min }=\frac{1}{\max _{x, \varepsilon}\left\{n(s) \cdot \sigma_{T}(\varepsilon)\right\}}
$$

When $\mathrm{n}(\mathrm{s})$ the background gas density is uniform in space, relation (11) becomes:

$$
\lambda_{\text {min }}=\frac{1}{n \cdot \sigma_{T, \max }}
$$

To obtain the distance covered without collision $S_{\text {vol, }}$ we put $1-P(S)$ $=R$, where $R$ is a random number between uniformly distributed 0 and 1 . Therefore, equation (10) becomes:

$$
S_{v o l}=-\lambda_{\min } \ln (1-R)
$$

After the free path $S_{v o l}$ the particle needs to collide with background gas target. This means if the covered distance $S$ obeys to this inequality: $S \geq S_{v o l}$. this collision can be real or fictitious. The collision type is determined from the following relations by considering the collision cross sections of the different real processes $\left(\sigma_{1}, \sigma_{2}, \ldots\right)$ and the fictitious or null collision $\sigma_{\text {null. }}$

$$
\left\{\begin{aligned}
\frac{1}{\lambda_{\text {min }}} & =n\left(\sigma_{1}+\sigma_{2}+\ldots+\sigma_{N}+\sigma_{\text {null }}\right) \\
& =\frac{1}{\lambda_{1}}+\frac{1}{\lambda_{2}}+\ldots+\frac{1}{\lambda_{N}}+\frac{1}{\lambda_{\text {null }}} \\
1 & =\frac{\lambda_{\text {min }}}{\lambda_{1}}+\frac{\lambda_{\text {min }}}{\lambda_{2}}+\ldots+\frac{\lambda_{\text {min }}}{\lambda_{N}}+\frac{\lambda_{\text {min }}}{\lambda_{\text {null }}}
\end{aligned}\right.
$$

The collision probability of each collision type is given by the following relations:

$$
P_{i}=\frac{\lambda_{\min }}{\lambda_{i}}=\frac{\sigma_{i}}{\sigma_{T, \max }}
$$

$$
\begin{cases}0<R \leq \frac{\sigma_{1}}{\sigma_{T, \max }} & \text { type } 1 \\ \frac{\sigma_{1}}{\sigma_{T, \max }<R \leq \frac{\sigma_{2}}{\sigma_{T, \max }}} & \text { type } 2 \\ ---------- & \\ \sum_{i=1}^{N} \sigma_{i} & \text { null collision }\end{cases}
$$

Once the collision type is determined, the velocity or the energy of the scattered particle can be calculated using the classical relation of total energy and momentum transfer verified during each collision as it is partly shown in the following section. After the collision, the free path of the next one is calculated from equation 10 .

\section{Simulation of the charged particle transport}

The plasma generated by low pressure RF discharge is a weakly ionized gas with homogeneous background density at gas temperature $\mathrm{Tg}=300^{\circ} \mathrm{K}$. The main charged particles in the case of $\mathrm{RF}$ low pressure discharge in argon are electrons $\left(\mathrm{e}^{-}\right)$and positive ions $\left(\mathrm{Ar}^{+}\right)$, the reactions in a simplified argon model are the following (Neyts et al., 2003; Wakayama and Nanbu 2003; Cenian et al., 2003; Phelps and Petrovic, 1999; Vahedi and Surenda, 1995). 


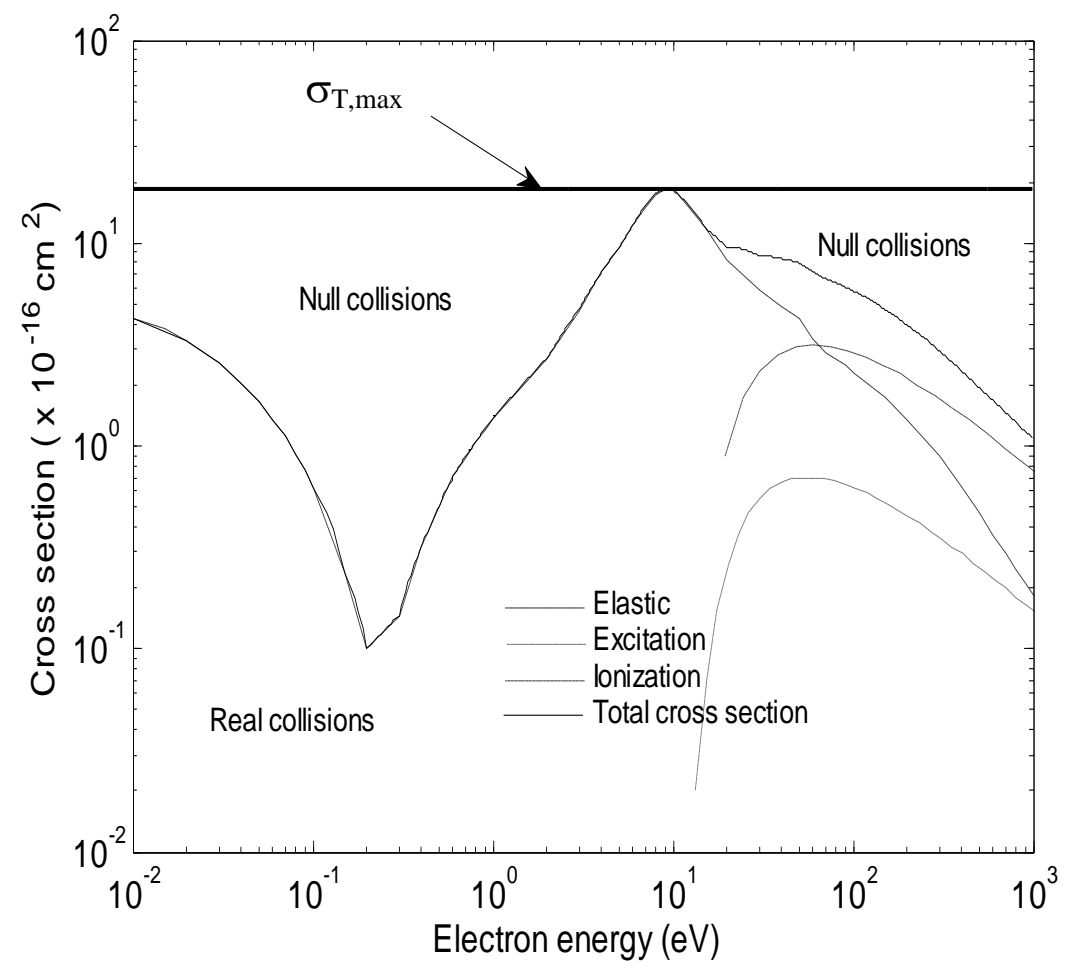

Figure 2. Electron-Argon collision cross sections.

(1) $e+A r \rightarrow e+A r$

(2) $e+A r \rightarrow e+A r^{*}$

(3) $e+A r \rightarrow 2 e+A r^{+}$

(4) $A r^{+}+A r \rightarrow A r^{+}+A r$

(5) $A r^{+}+A r \rightarrow A r+A r^{+}($Charg e Exchange $)$

\section{Electron-Argon collisions}

The considered electron-neutral cross sections are taken from Phelps and Petrovic (1999) and are displayed in Figure 2. In the case of the electron-atom elastic collisions, the incident electron of energy $\varepsilon_{0}$ scatters through an axial scattering angle $\chi$ and azimuthal scattering angle $\psi$. These angles are defined by two random numbers $\left\{R_{1}, R_{2}\right\} \in[0,1]$ (Vahedi and Surenda, 1995).

$\cos \chi=\frac{2+\varepsilon-2(1+\varepsilon)^{R 1}}{\varepsilon}$

$\psi=2 \pi R_{2}$

Once the axial scattering angle is determined, we can calculate the energy loss of the electron during an elastic scattering event.

$$
\Delta \varepsilon=2 \frac{m}{M}(1-\cos \chi) \varepsilon_{0}
$$

$m$ is the electron mass and $M$ is the mass of the neutral atom. Although this energy loss is small because of the low mass ratio, it is only energy loss mechanism for the low energy electrons with energies less than excitation and ionization thresholds. In argon discharge, this energy loss is not very effective because of the presence of Ramsauer minimum in the elastic scattering cross section. After an excitation processes, a part of the incident electron energy is transformed to the internal states of the argon atom. In the model all internal states are merged into one single excitation level with energy of $\varepsilon_{e x c}=11.5 \mathrm{eV}$. After an excitation collision event, the scattering angles are determined from relations (Equation 17). If the kinetic energy of electron exceeds the ionization threshold energy, ionization collision can occur leading to the creation of pair of an electron-ion. Therefore, after an ionization event, there are two electrons (ejected and scattered ones) and an ion (reaction 5). Because of the small electron-to-ion mass ratio, we can assume that the momentum of the scattered electron is much lower less than the momentum of the neutral. In other words, the incident electron strips an electron of the neutral, and the neutral becomes ion. The direction of each electron is determined by the following relation (19). The residual kinetic energy of the incident electron after ionization will be divided between the two electrons according to the following relations (Vahedi and Surenda, 1995).

$$
\left\{\begin{array}{l}
\varepsilon_{e j}=R \cdot \frac{\left(\varepsilon_{0}-\varepsilon_{i o n}\right)}{2} \\
\varepsilon_{s c}=\varepsilon_{0}-\varepsilon_{i o n}-\varepsilon_{e j}
\end{array}\right.
$$

$R$ is random number uniformly distributed between 0 and $1, \varepsilon_{0}$, $\varepsilon_{i o n}$, $\varepsilon_{e j}, \varepsilon_{s c}$ are respectively the energies of the incident electron just 


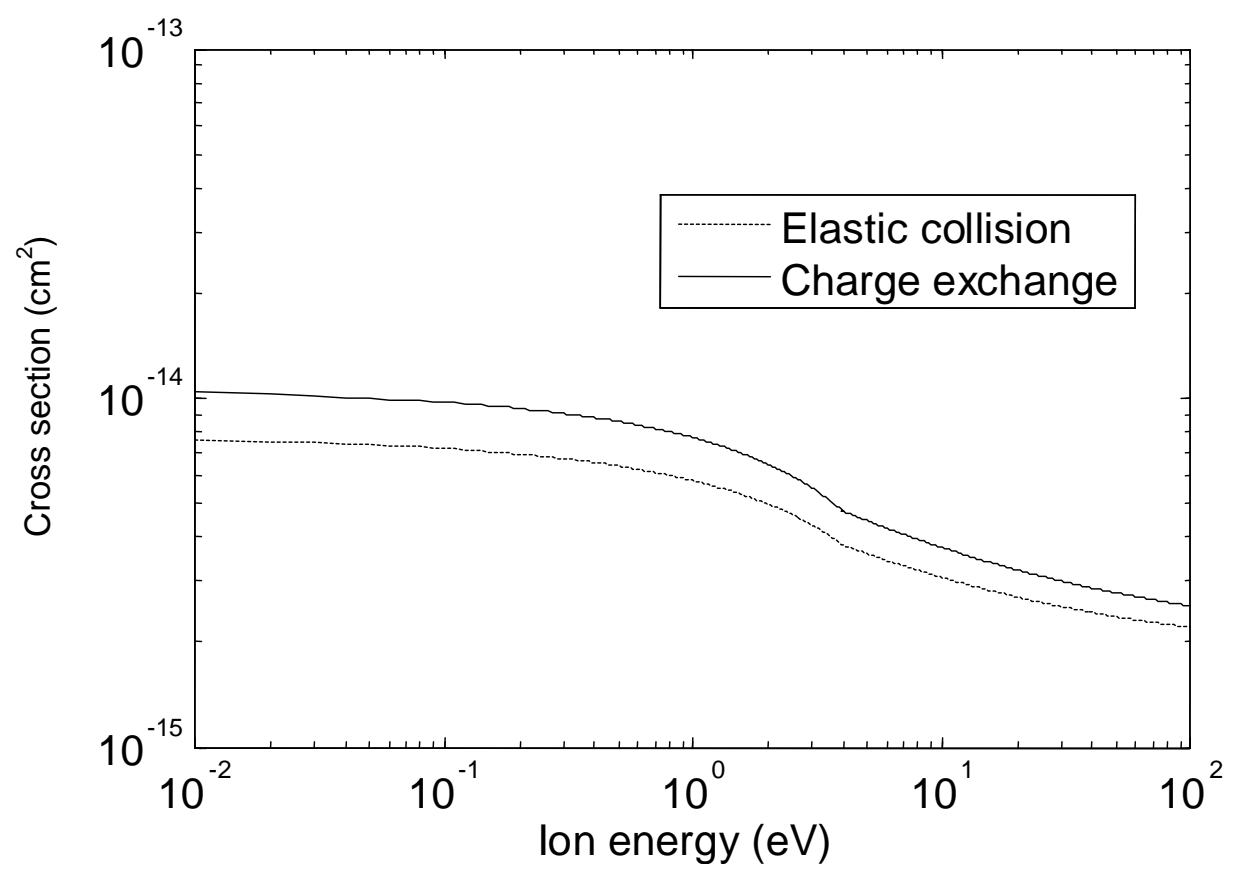

Figure 3. $\mathrm{Ar}^{+}-\mathrm{Ar}$ collision cross sections.

before the collision, the ionization threshold, the electron ejected and the scattered electron.

\section{Ion-Argon collisions}

Figure 3 shows the ion-neutral collision cross sections used in this model which are taken from Vahedi and Surenda. (1995).

Due to the masses of ion and atom are which are almost the same, the energy exchange during $\mathrm{Ar}^{+}-\mathrm{Ar}$ collision is much more important than the electron case. So, this has to be correctly taken into account. The collision is treated in the reference of the centre of mass. The velocities of target neutrals are sampled from a Maxwellian velocity distribution at room temperature $\left(\mathrm{Tg}=300^{\circ} \mathrm{K}\right)$. The axial scattering angle $\chi$ is assumed isotropic as done by Vahedi and Surenda. (1995) and the azimuthal scattering angle $\psi$ is distributed uniformly between $[0,2 \pi]$. These angles are defined by two new random numbers $\left\{\mathrm{R}_{1}, \mathrm{R}_{2}\right\} \in[0,1]$.

$$
\left\{\begin{array}{l}
\cos \chi=\sqrt{1-R_{1}} \\
\psi=2 \pi R_{2}
\end{array}\right.
$$

After an elastic collision between ion and atom, the energy of the incident ion becomes:

$$
\varepsilon_{\text {proj }}=\varepsilon_{0} \cos ^{2} \chi
$$

$\varepsilon_{\text {proj }}$ and $\varepsilon_{0}$ are respectively, the energies of the ion just after and just before the collision. After a charge exchange (or charge transfer) collision, an electron is assumed to hop from the neutral onto the ion, causing the incident ion neutralization and the neutral target becomes an ion. The new ion leaves the collision with the velocity of the incident neutral, and the new neutral takes the velocity of the incident ion.

\section{RESULTS AND DISCUSSION}

In order to show the validity of our model, our calculations are compared to the experimental results of Hopkins (1995) that has measured electron density using two different techniques: a direct measurement using an interferometer and an indirect measurement where the electron density is estimated through the ion density with a correction factor. Figure 4 shows the comparison between the calculated electron densities in the middle of the discharge for two gas pressures $(6.66$ and $13.33 \mathrm{~Pa})$ and $400 \mathrm{~V}$ for the peak-to-peak RF voltage at $13.56 \mathrm{MHz}$. The calculated results remain inside the relative measurement error $( \pm 20 \%)$. Figure 5 also shows the comparisons between our calculations at $13.33 \mathrm{~Pa}$ for the gas pressure and various RF peak voltages $(150,200,250$ and $300 \mathrm{~V}$ ) and for a frequency of $13.56 \mathrm{MHz}$ and the experimental measurements of Hopkins (1995). The observed good agreement confirms the good quantitative behavior of our RF discharge model. Figure 6 shows the spatial variation of electron densities obtained after a large number several RF cycles. This comparison is done in the case of two methods of collisions treatments. The first one is based an optimized calculation of collision free path adopted in this work and the second one is based on the free time of flight using both the classical null collision technique. The results obtained from the two methods of collision treatment are in a very good agreement. However, the computing time lower by about $10 \%$ in the case of the particle code using the free path method. 


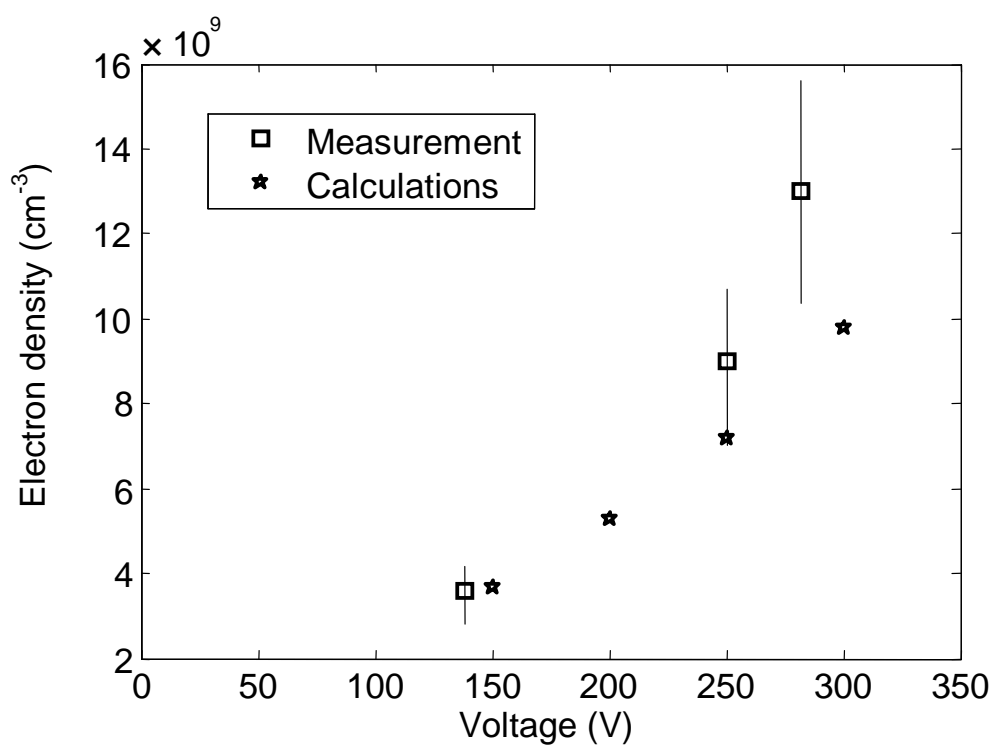

Figure 4. Comparison as a function of RF voltage between the calculations and the measurements of Hopkins (1995) at $13.33 \mathrm{~Pa}$ for the gas pressure at $13.56 \mathrm{MHz}$.

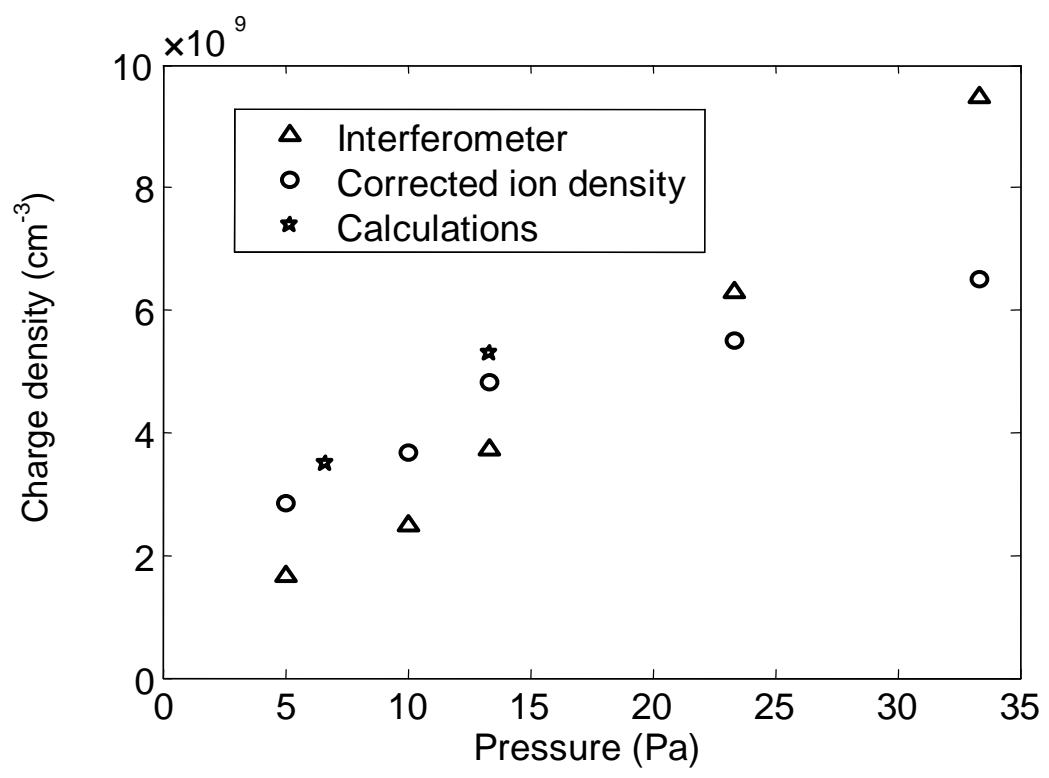

Figure 5. Comparison as a function of gas pressure between calculations and the measurements of Hopkins (1995) obtained following two experimental methods (interferometer and correction ion density) under 200V for the peak of the RF voltage at $13.56 \mathrm{MHz}$.

Table 1 shows a comparison between calculations and measurements of the RF electrical discharge characteristics (current and power) for different operating conditions. The calculation data corresponds to the amplitudes of the fundamental Fourier components, and the experimental data of the current are taken from
Overzet (1995). The calculated current density is lower than the measured one, but it is in the same order of magnitude. This expected discrepancy is due to the effect of geometry which is not considered in the present dimensional model.

After validation of the model, we analyze in the 


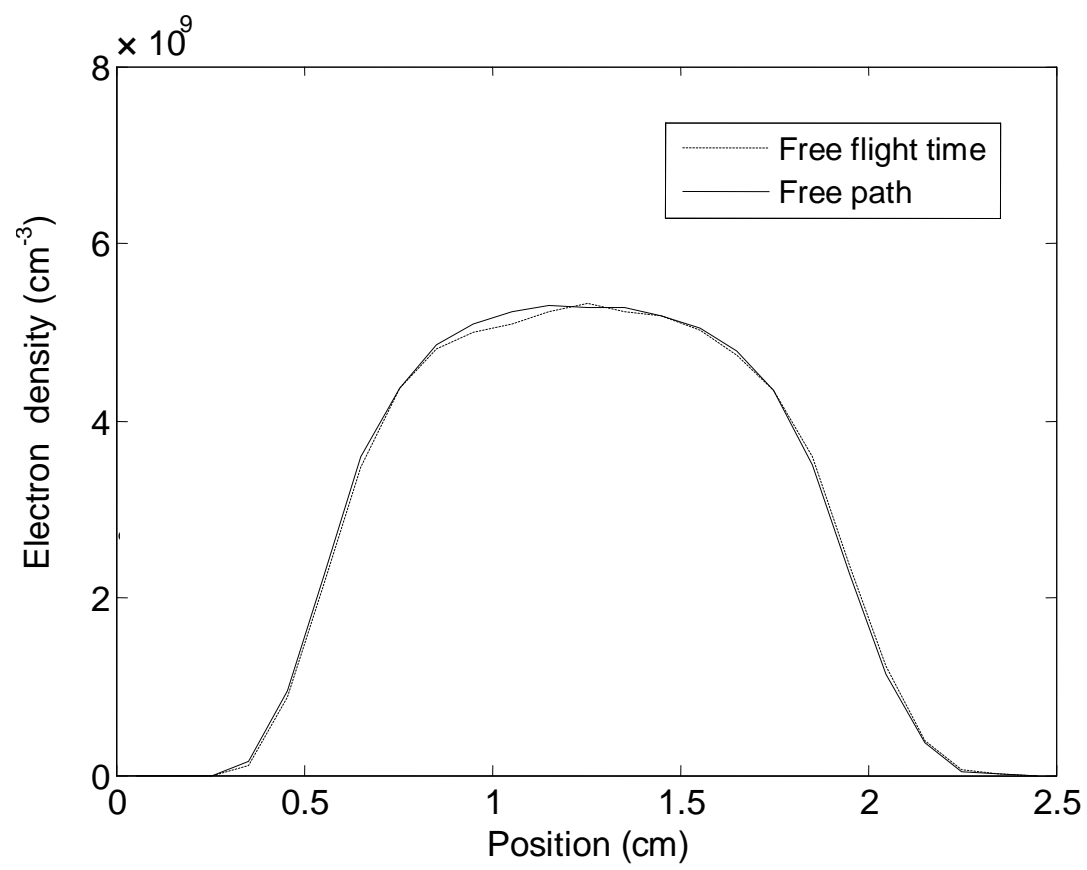

Figure 6. Comparison along the discharge axis between the results obtained from particle model using free flight time and free path methods for the collision treatment in the case of a RF voltage of $200 \mathrm{~V}$ at $13.56 \mathrm{MHz}$ and $13.33 \mathrm{~Pa}$ of $\mathrm{Ar}$ pressure.

Table 1. Electrical characteristics.

\begin{tabular}{lccc}
\hline Variable & Pressure $(\mathbf{P a})$ & Current $\left(\mathbf{m A} / \mathbf{c m}^{2}\right)$ & Power $\left(\mathbf{m W} / \mathbf{c m}^{2}\right)$ \\
\hline \multirow{2}{*}{ Model } & 6.66 & 1.60 & 3.20 \\
& 9.99 & 1.80 & 4.60 \\
& 13.33 & 2.10 & 6.20 \\
Experiment & 13.33 & & $\sim$ \\
\hline
\end{tabular}

following some electrical characteristics of the RF discharge. Figure 7 shows the spatiotemporal variation of the plasma potential in the discharge during the RF cycle. In the plasma region (middle of the discharge) the potential is always higher than those of electrodes which is an effective barrier required to maintain the stability and the electric neutrality of the plasma. In fact, this average value is close to the half value of the peak voltage applied because the self-bias voltage is equal to zero in the case of a symmetrical discharge. In the edge of plasma sheath on the plasma region, Figure 8 clearly shows the strong gradient of ion density which reflects the large number of ionizing collisions occurring in this region and due to the memory effect of the high magnitude of electric field in the sheath region near the electrodes. Figure 9 shows that, unlike the ions, the electrons arrive to follow quasi-instantaneously the variations of the electric field due to their low inertia. At the beginning of the RF cycle, the electrons are attracted towards the right electrode (momentarily an anode) giving a negative space charge which reduces the electric field in the vicinity of this electrode and causes the contraction of the sheath. Then during the evolution of the RF cycle, the electrons are repelled towards the plasma region (middle of the discharge) which leads to a positive space charge in the sheath. This appearance of a positive space charge in the vicinity of the electrode (momentarily a cathode), leads to the increase of the electric field at this region causing the expansion of the sheath. So the movement of electrons covering and uncovering the positive space charge in the sheaths is the basis of the formation and the oscillation of the sheaths. The contraction and expansion of the sheaths themselves are the basis of the periodic electron motion. It is a mutual 
Benyoucef et al. $\quad 5263$

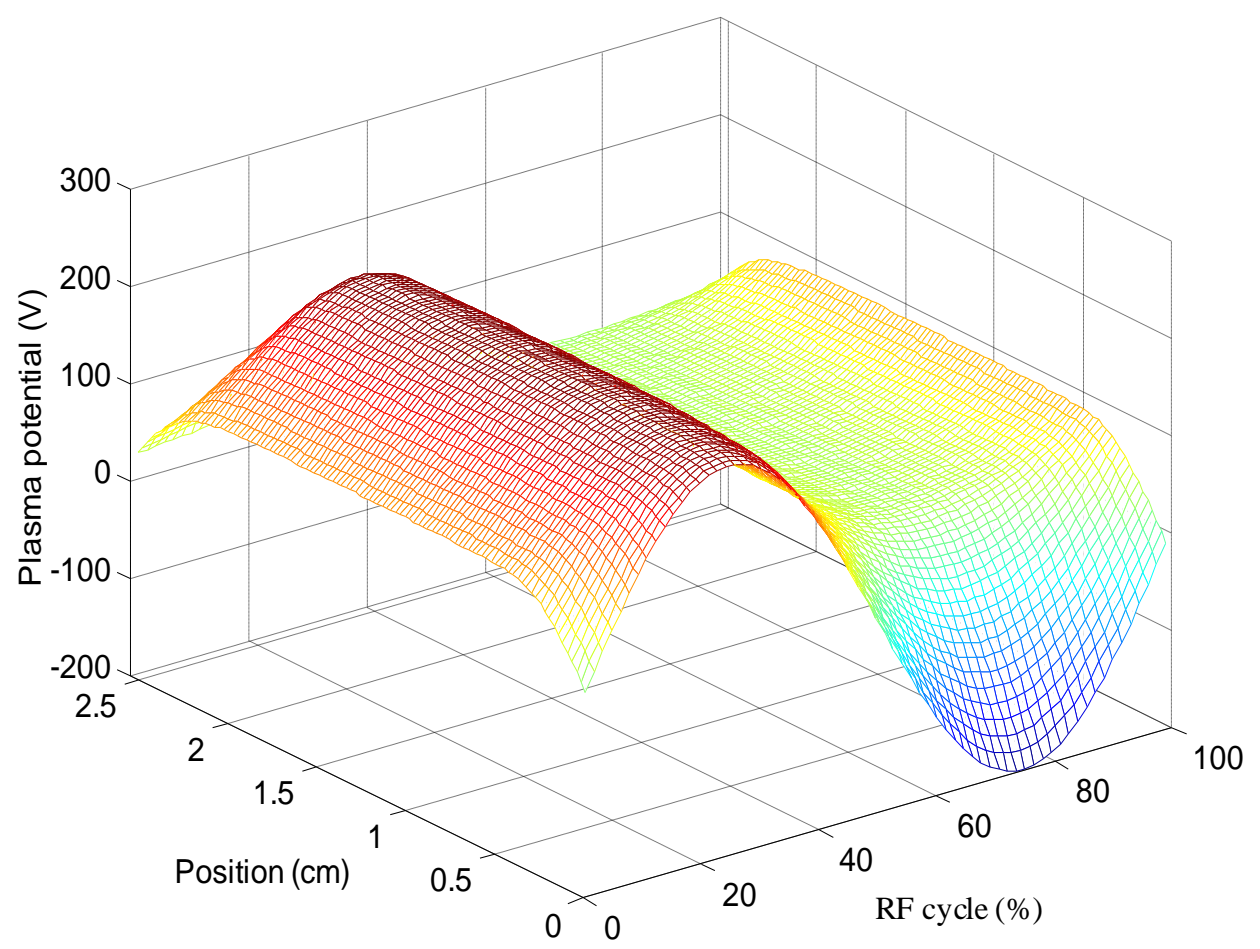

Figure 7. Spatiotemporal variation of the plasma potential for $13.33 \mathrm{~Pa}, 200 \mathrm{~V}, 13.56 \mathrm{MHz}$ and a gap distance of $2.54 \mathrm{~cm}$.

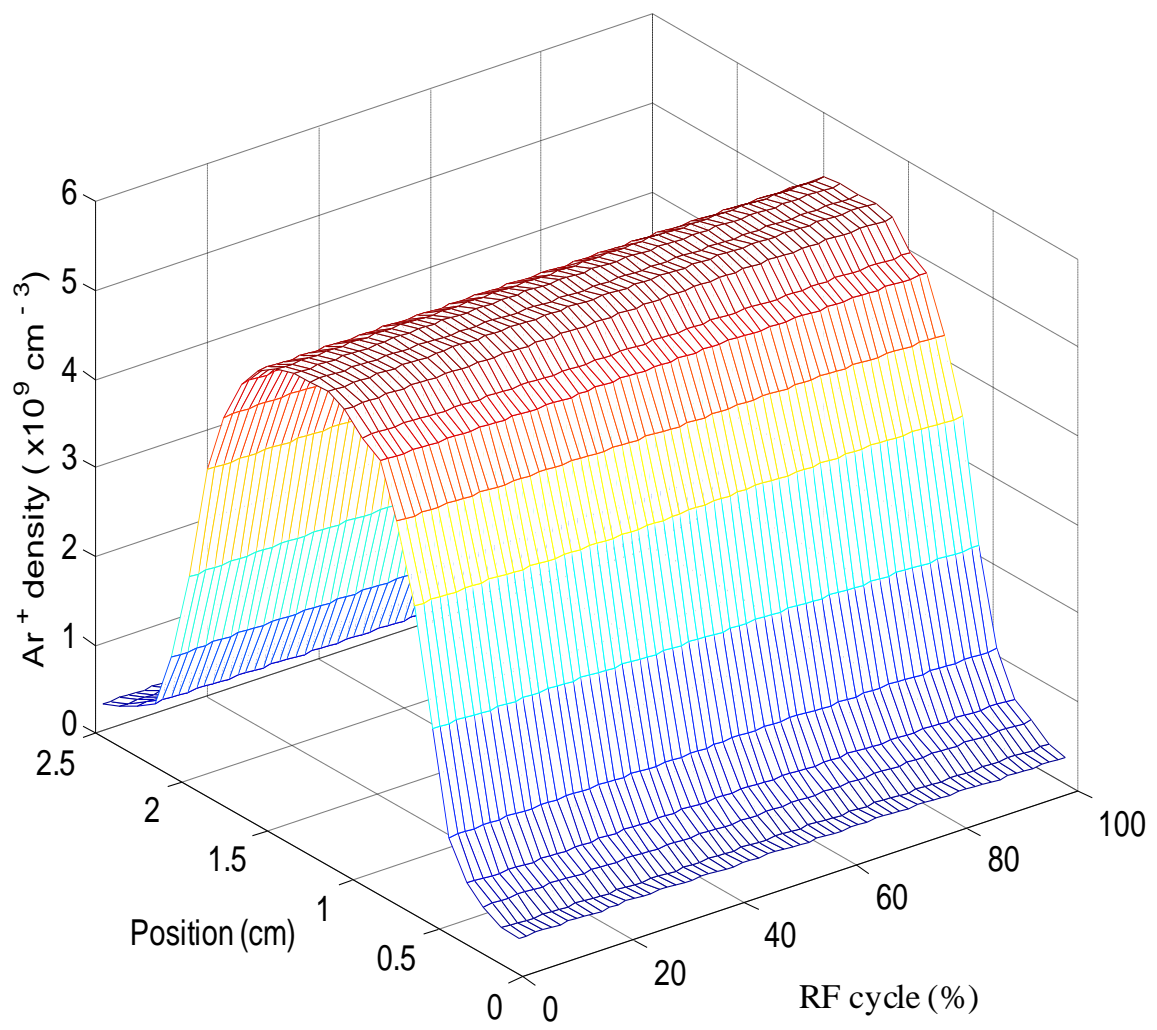

Figure 8. Spatiotemporal variation of $\mathrm{Ar}^{+}$density for $13.33 \mathrm{~Pa}, 200 \mathrm{~V}, 13.56 \mathrm{MHz}$ and a gap distance of $2.54 \mathrm{~cm}$. 


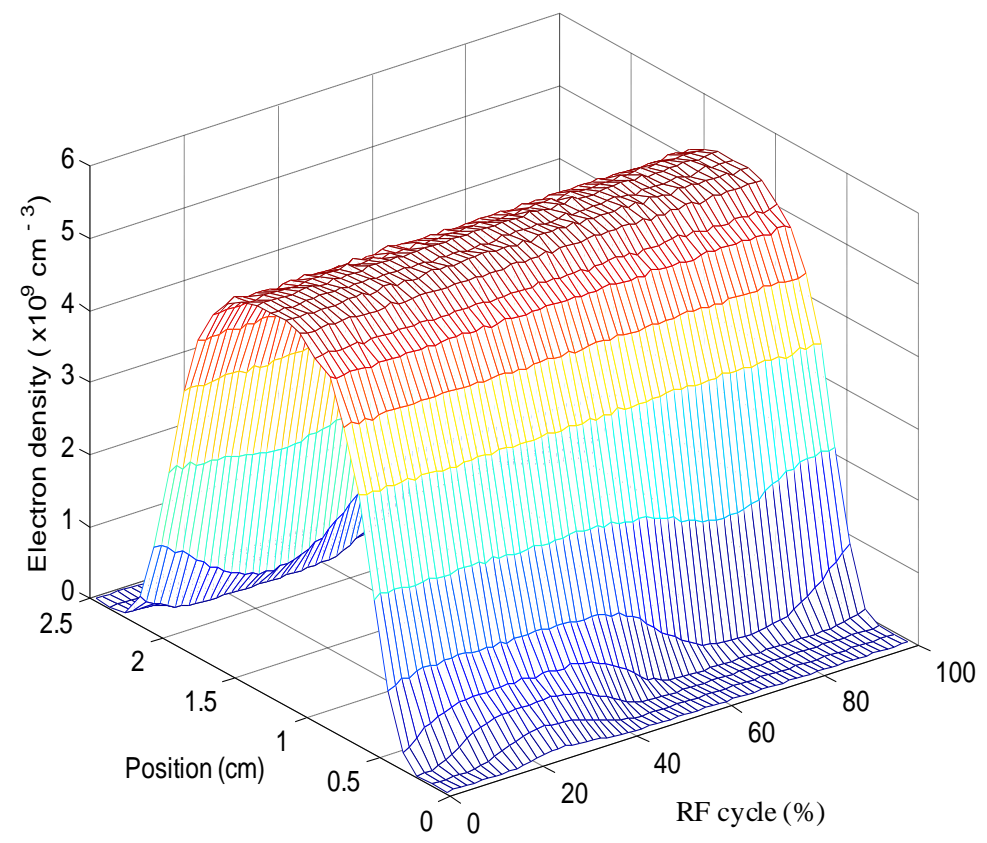

Figure 9. Spatiotemporal variation of the electron density for $13.33 \mathrm{~Pa}$, $200 \mathrm{~V}, 13.56 \mathrm{MHz}$ and a gap distance of $2.54 \mathrm{~cm}$.

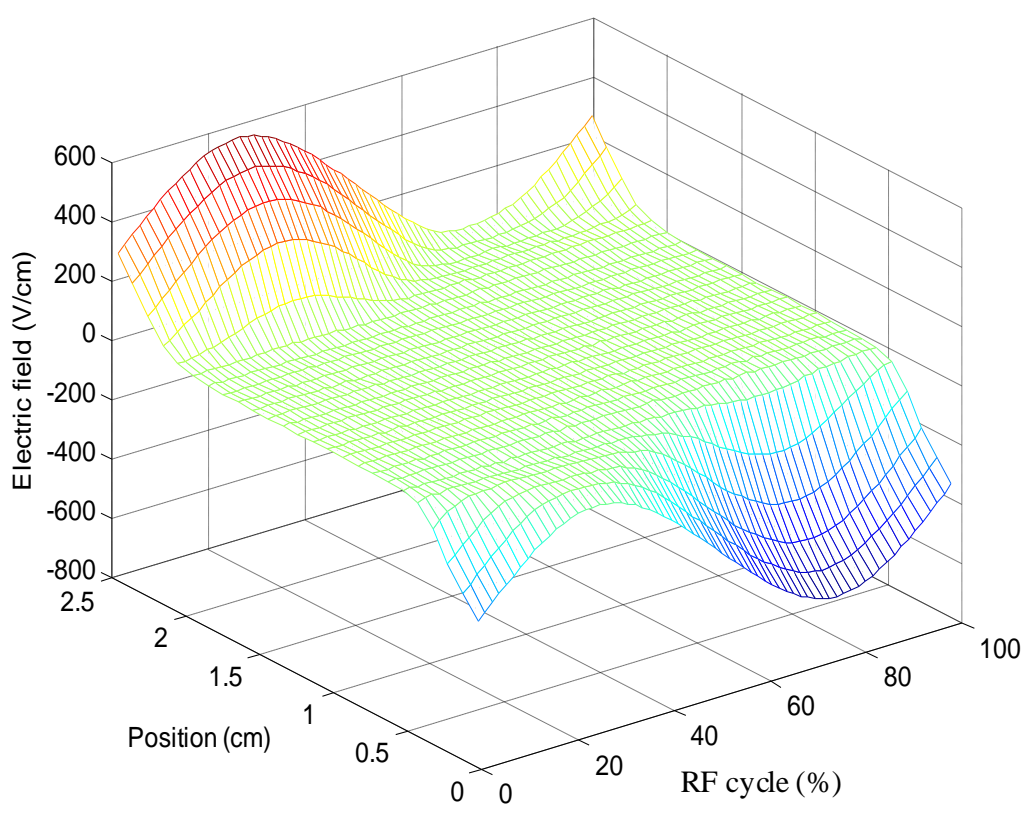

Figure 10. Spatiotemporal variation of the electric field for $13.33 \mathrm{~Pa}, 200 \mathrm{~V}$, $13.56 \mathrm{MHz}$ and a gap distance of $2.54 \mathrm{~cm}$.

effect which couples strongly the electron transport and the variation of the electric field.

Figure 10 displays the spatiotemporal variation of the electric field depending on the position and different times of the RF cycle. The electric field is very low in the plasma because of the electrical neutrality while it is very high in the sheath regions for the reasons already mentioned concerning the movement for reasons already emphasized concerning the oscillatory motion of electrons around quasi-static ions. At the electrodes, the electric field which is by definition the potential gradient follows, as expected, the sinusoidal variation at the 
same frequency of the applied RF voltage. Indeed, in our case the time evolution of the applied RF voltage Vrf (t) with a maximum value of $200 \mathrm{~V}$ obeys to this relation:

$\operatorname{Vrf}(t)=200 \cdot \sin (2 \pi f)(f=13.56 \mathrm{MHz})$

Vrf $(t)$ is applied to the powered right electrode $(x=0$ $\mathrm{cm})$ while the left electrode $(x=2.54 \mathrm{~cm})$ is grounded.

\section{Conclusion}

The PIC MC model based on the free path for collisions treatment developed in this work is validated from the excellent agreement with experimental data taken from the literature in the case of RF discharge at low pressure argon background gas. The use of free path method allows for saving about more than $10 \%$ of computation time in comparison to the PIC MC method based on the usual free time of flight. Then, the plasma region in the middle of the discharge and the sheath region near the powered and grounded electrodes have been analyzed from the spatiotemporal variations of charged particle densities and space charge electric field thus showing the classical expansion and contraction of the plasma during a RF cycle.

\section{REFERENCES}

Brennan MJ (1991). Optimization of Monte Carlo codes using null collision techniques for experimental simulation at low E/N. IEEE Trans. Plasma Sci. 2:256-261.

Cenian A, Chernukho A, Leys C (2003). Particle-In-Cell Monte Carlo (PIC-MC) simulations of plasma-wall interaction in low-pressure AR plasma. Radiation Phys. Chem. 1-2:109-113.

Hellegouarch F, Arefi-Khonsari F, Planade R, Amouroux J (2001). PECVD prepared $\mathrm{SnO} 2$ thin films for ethanol sensors. Sensors Actuators B 73:27-34.

Hess DW (1986). Plasma-Surface Interactions in Plasma-Enhanced Chemical Vapor Deposition. Ann. Rev. Mater. Sci. 16:163-183.

Hopkins MB (1995). Langmuir probe measurements in the gaseous electronics conference RF reference cell. J. Res. Nat. Institute Standards 4:415-425.

Kawamura E, Bridsall CK, Vahedi V (2000). Physical and numerical methods of speeding up particle codes and paralleling as applied to RF Discharges. Plasma Sour. Sci. Technol. 3:413-428.

Koura K (1986). Null-collision technique in the direct-simulation Monte Carlo method. Phys. Fluids 11:3509-3511.
Nanbu K (1994). Simple method to determine collisional event in Monte Carlo simulation of electron-molecule collision. Japanese J. Appl. Phys. 8:4752-4753.

Neyts E, Yan M, Bogaerts A, Gijbels R (2003). PIC-MC simulation of an RF capacitively coupled $\mathrm{Ar} / \mathrm{H}_{2}$ discharge. J. Appl. Phys. 3:50255033.

Overzet LJ (1995). Microwave diagnostic results from the gaseous electronics conference RF reference. J. Res. Nat. Inst. Stand. Technol. 4:401-414.

Phelps AV, Petrovic ZL (1999). Cold-cathode discharge and breakdown in argon: surface and gas phase production of secondary electron. Plasma Sources Sci. Technol. 3:4752-4753.

Sandu I, Presmanes L, Alphonse P, Tailhades P (2006). Nanostructured cobalt manganese ferrite thin films for gas sensor application. Thin solid Films 495:130-133.

Skullerud HR (1968). The stochastic computer simulation of ion motion in gas subjected to constant electric field. J. Appl. Phys. 11:15671568.

Sugawara H, Naoki Mori, Yosuke Sakai, Yoshiyuki S (2007). Timesaving techniques for decision of electron-molecule collisions in Monte Carlo simulation of electrical discharge. J. Comput. Phys. 1:298-304.

Vahedi V, Surenda M (1995). A monte Carlo collision model for the particle-in-cell method: application to argon and oxygen discharge. Comput. Phys. Commun. 1-2:179-198.

Van der Velden MHL, Brok WJM, Van der Mullen JJAM, Goedheer WJ, Banine V (2006). Particle-in-cell Monte Carlo simulations of an extreme ultraviolet radiation driven plasma. Phys. Rev. 3:1-6.

Wakayama G, Nanbu K (2003). Study on the dual frequency capacitively coupled plasmas by the Particle-In-Cell/Monte Carlo method. IEEE Trans. Plasma Sci. 4:638-644.

Wu BT, Xiao DM, Liu ZS, Zhang LC, Liu XL (2006). Analusis of insulation characteristics of C-C4F8 and N2 gaz mixtures by the Monte Carlo method. J. Phys. D: Appl. Phys. 19:4204-4207.

Yousfi M, Hennad A, Alkaa A (1994). Monte Carlo simulation of electron swarms at low reduced electric fields. Phys. Rev. 4:32643273. 